\title{
De la marginación a la inclusión social: Ley de discapacidades
}

\author{
Marcos Moreira Argudo / Alberto Paredes Ochoa
}

\section{RESUMEN}

Debido a la marginación de la sociedad, la inclusión laboral del discapacitado se origina por exigencias del Gobierno con acción del Ministerio de Trabajo y Empleo, amparadas estas a partir de su publicación en el Registro (Oficial Nro.198. Actualmente el Código de Trabajo del Ecuador establece que cada empresa con un número mínimo de veinticinco trabajadores debe hacer una inclusión de, por lo menos, una persona con discapacidad.

Inte esta estructura, habria que cuestionarse, ¿qué tan pertinente es la política pública establecida en todas nuestras leyes, alrededor de la inclusión laboral de personas con discapacidad en el Ecuador? Por lo que se analizará, el pro y el contra que representa la Ley Orgánica de Discapacidades del Ecuador al respecto y su influencia en el marco legal ecuatoriano, especialmente en lo laboral.

\section{PALABRAS CLAVE}

Discapacidad, Inclusion Laboral, derechos, trabajadores, inclusión social.

\section{ABSTRACT}

The Ecuadorian Ministry of Work and Employment outlaws discrimination and requires the inclusion of the disabled in the labor force. This was established in ()fficial Gazctte No. 198. $\Lambda$ ccording to the Labor Code, companies with 25 or more employees must hire at least one disabled person. Given this structure, one wonders whether the legislation concerning the inclusion of the disabled is effective.

This paper will analyze the pros and cons of the Ecuadorian Law on Disabilities and its influence on Ecuador's legal framework, particularly in connection with the inclusion of the disabled in the labor force.

\section{KEYW/ORDS}

Disability, Labor Inclusion Labor, rights, morkers, social inclusion. 


\section{Introducción}

Como todo proceso constructivo en la historia ecuatoriana, debieron suceder acontecimientos impactantes para que la sociedad se haga copartícipe de las necesidades de cambios, esto se debe a que las personas cuando se ven inmersas en las problemáticas que le ocurren a ciertos sectores sociales, sean estas por abusos, marginación, enfermedades, pérdidas de todo tipo, hacen germinar la concientización de establecer premisas que sean discutidas a todo nivel, con este accionar comienzan a influir a todos los entes directa o indirectamente relacionados para que sean elevados a lẹ:

Fsto permitirá establecer el siguiente objetivo general del trabajo que se presenta: valorar los avances que se están realizando en el Ecuador en materia de inclusión social al ser discapacitado.

Para ello se plantean como objetiros especificos:

- Determinar antecedentes sobre el aspecto de ser discapacitado.

- Analizar las normas juridicas para su inclusión laboral.

- Analizar las normas generales que priorizan la inclusión social.

\section{Desarrollo}

Las personas nacen, crecen $y$ se desarrollan en diferentes ámbitos, cada ser logra expandir sus capacidades, habilidades, competencias para adaptarse a la sociedad donde se desenvuelve. Claro está que la naturaleza tan incierta hace que ciertos seres no logren un desarrollo corporal normal o en su caso, por situaciones fortuitas, formaron parte de lamentables accidentes.

Pero eso no significa que este grupo humano no pueda cultivar sus talentos, ejemplos de rida son:
- Louis Braille (Discapacidad visual)

- Jaime de Borbón (Discapacidad auditiva)

- I.ou Ferrigno (Discapacidad auditiva)

- Marlee Matlin (Discapacidad auditiva)

- Ludwig Van Beethoren (Discapacidad auditiva)

- Christopher Reere (Discapacidad motriiz)

- Belén García Bellón (Discapacidad físico motora)

- Vincent V'an Gogh (Discapacidad psicosocial)

- Indrea Bocelli (Discapacidad visual)

- Francisco de Gora (Discapacidad auditiva)

- José Feliciano (Discapacidad visual)

- Rick Allen (Discapacidad físico- motora)

- Steric Wonder (Discapacidad visual)

- Stephen Hawking (Discapacidad motora)

- Frida Kahlo (Discapacidad músculo esquelética)

- John Nash (Discapacidad psicosocial)

- Gabricla Brimmer (Discapacidad físico motora)

- Anne Sullivan (Discapacidad visual)

- Tony Meléndez (Discapacidad físico motora)

- Mdrián Hernández (Discapacidad físico motora)

- Ray (harles (Discapacidad risual)

- Mýdeé Ramírez. (Discapacidad físico motora)

- Liu Wei (Discapacidad físico- motora)

- Tommy Lommy (Discapacidad físico motora) (CON $A$ PDIS)

Por ende se hace necesario un cambio de conciencia en la parte humana, una adaptación de las estructuras para brinctarles faciliclades en su libre morimiento, normas juridicas para establecer parámetros que incentiven su inclusión y una sociedad más justa con todos sus ciudadanos. 
In el 2008, al estado de ser discapacitado, se lo denominaba minusválido (Congreso Nacional de la República del Ecuador; 1992, página 70) y la perspectiva para estas personas en el mercado laboral era incierta (Hoy Quito, 2007, 27 de diciembre). De ahí en adelante, a lo largo de estos años la ejecución de la l.ey. (Orgánica de Discapacitados en el área laboral de las persona con condiciones discapacitantes o discapacitados, le ha dado un giro muy loable a la vida de estas, permitiéndoles un espacio dentro de una organización empresarial pública o privada, logrando de esta forma una visión más sensible de la sociedad en general, por lo que a partir de entonces nuestro pais comenzó a vivir un Ecuador sin barreras.

Iin este sentido, las personas con discapacidad, en mayor o menor grado, se constituyen en un sector discriminado, principalmente cuando la condición de discapacidad se le suma la condición de pobreza.

Esto puede generar bajos niveles de autoestima, poca independencia y autonomia, o ninguna participación de los procesos políticos, sociales y económicos de la sociedad, incluido en este aspecto el establecimiento y desarrollo de personas con discapacidad. (Montero, 2005 p 6)

En los últimos 60 años, ha habido una profunda reformulación de los derechos ! habilidades de las personas con discapacidad; sin embargo, en $A$ mérica Latina, las perspectivas institucionales $y$ las respuestas programáticas no siempre han ido de la mano con la evolución política global (Bubbinic, Maz7a, Pungiluppi, Massiah, 2004, pag.67)

En Ecuador, estos acontecimientos han hecho que para el año 2009 se genere la obligación de la inclusión laboral del 4\% de personas con discapacidad en las nóminas totales empresariales a partir de los 25 colaboradores, y bajo un mismo empleador.
Ese es el punto de partida que hace al ser humano un ente con conciencia. I a Rill la define como en la primera acepción, propiedad del espíritu humano de reconocerse en sus atributos esenciales y en todas las modificaciones que en sí mismo experimenta y la DEA la define en la primera acepción, conocimiento inmediato y espontáneo de una realidad. (Alles, 2005, página 80) Generándose dos clasificaciones de la misma: la conciencia individual y la conciencia colectiva.

Por lo expuesto sobre conciencia se describirá cada una de ellas en dos ámbitos diferentes, psicológico y laboral, permitiendo que desde una perspectira psicológica la conciencia individual sea la experiencia humana de multiplicidad de cosas que son separadas (Pintos, 1996, página 34). Ahora bien se plantea una hipótesis que desde una perspectiva laboral la conciencia individual sea la forma en la que el grupo prioritario abra espacios laborales retadores.

Analizado el primer punto, se plantea desde una perspectiva psicológica la conciencia colectiva parte de un grupo social más o menos grande, cuỵas representaciones son compartidas por sus miembros (Doron, 2008, página 118). Mhora bien se plantea una hipótesis que desde una perspectiva laboral la conciencia colectiva sea cuando el grupo humano se prepara psicológicamente para ser acogidos, sin problema alguno, en las empresas.

Por estas razones se llegó a establecer en el Ecuador los primeros indicios de cambios de conciencia, la Asamblea Constituyente al redactar la Constitución del 2008 promulgó en su Artículo 47 que "El Estado garantizará políticas de prevención de las discapacidades y; de manera conjunta con la sociedad y la familia, procurará la equiparación de oportunidades para las personas con discapacidad y su integración social" buscando dignificar 
laboral y socialmente a los discapacitados que están aptos en edad y preparación técnica diversa $y$ dar paso al cumplimiento de la Reforma Laboral en favor de la inclusión de los mismos. $Y$ es así que se logró cerrar el periodo fiscal 2013 con la contratación y permanencia contractual fija en 7679 instituciones, entre públicas y privadas.

Pero el Ministerio de Relaciones Laborales: Dirección de $\lambda$ tención a los grupos prioritarios, realizó un censo a 9004 empresas, censadas en las diversas provincias del país. Dando como resultado de esta cifra; 1325 instituciones entre privadas y públicas no cumplen con lo mandatorio. En la realidad existen contratos dolosos que se lograron descubrir al inicio de las auditorías de investigación, entre los años 2009-2010, que en su momento fueron motivos de escándalos, así como el pago de sanciones económicas, las mismas cuyo cálculo resulta de la multiplicación de la base del salario mínimo.

Huxley, 1994 sostiene que la conciencia es fundamental y su desarrollo es la meta principal de la existencia, (Grof, 2003, página 21) generándose dos clasificaciones de la misma: la conciencia individual y la conciencia colectiva.

Analizándose por separado, dejará claro sus diferencias o similitudes según sea el caso de las estructuras conceptuales que posee cada término. Se describirá cada una de ellas en dos ámbitos diferentes: psicológico y laboral.

El primero permite que desde una perspectiva psicológica la conciencia individual sea dada como la experiencia humana de multiplicidad de cosas que son separadas (Pintos, 1996, página 34) y desde una perspectiva laboral se sugiere que la conciencia individual es la forma en que el grupo prioritario abre espacios laborales retadores.
Fil segundo permite que desde una perspectiva psicológica la conciencia colectiva parta de un grupo social más o menos grande siendo las representaciones comparticlas por sus miembros (Doron, 2008, página 118) y desde una perspectiva laboral se sugiere que la conciencia colectiva, es cuando el grupo humano se prepara de manera psicológica para acogerlos sin problema alguno en las empresas.

En Ecuador los primeros indicios de cambios de conciencia, buscaron dignificar laboral y socialmente a los discapacitados que están aptos en edad y preparación técnica diversa a dar paso al cumplimiento de la Reforma I aboral en favor de la inclusión de los mismos, y es así que se logró cerrar el período fiscal 2013 con la contratación y permanencia contractual fija en 7679 instituciones, entre públicas y privadas de 9004 censadas en las diversas provincias del país, de acuerdo al censo de control por parte del Ministerio de Relaciones Laborales: Dirección de $A$ tención a los grupos prioritarios. Esta cifra indica que 1325 instituciones entre privadas y públicas no cumplen con lo mandatorio. Existen en la realidad contratos dolosos que se lograron descubrir al inicio de las auditorías de investigación entre los años 2009-2010, que en su momento fueron motivos de escándalos, como también el pago de sanciones económicas: las mismas cuyo cálculo resulta hasta hoy de la multiplicación de la base del salario mínimo unificado vigente por diez por el número de discapacitados que faltaren para el $4 \%$ de la plantilla nominal total. Ante ello, la infracción de la ley implicaría para las empresas más que un mandato de riesgo laboral, también un fuerte gasto económico que no justificaba para que sea incurrido, esto ratificaría la exigencia de toda una programación estratégica para cumplirla. Esta organización que abarcaría desde el re-diseño de las estructuras físicas e instalaciones, procedimientos en el área de los Recursos Humanos, imple- 
mentaciones de mediclas de Seguridad Industrial! Medicina Preventiva; el paso estaba dado, las Auditorías de Control enseñaban el camino por donde seguir sin perjudicar a nadie, ni apuntar prejuicios, el Ecuador sin Barreras ameritaba cambios significativos en los procesos organizacionales, los cuales ventajosamente no demoraron en implementarse, los integrantes de las mismas abiertos al cambio lo hicieron, los mismos se ejecutan hasta nuestros días.

İn un inicio los empresarios se cuestionaban las percepciones para definir a la persona discapacitada y captar con ello ese talento humano con esas condiciones, pero su concepto lo encontramos en el $\lambda$ rt.7 de la ley sujeta de este análisis: "Se entiende por persona con deficiencia o condición discapacitante a toda aquella que, presente disminución o supresión temporal de alguna de sus capacidades físicas, sensoriales o intelectuales, manifestándose en ausencias, anomalías, defectos, pérdidas o dificultades para percibir, desplazarse, oír y/o ver, comunicarse, o integrarse a las actividades esenciales de la vida diaria, limitando el desempeño de sus capacidades: y; en consecuencia el goce y ejercicio pleno de sus derechos". Esto llevó a que los profesionales de la rama de los Recursos Humanos, que ahora se les denomina de Talento Humano o de Gestión Humana; se ampararan en este artículo para que con esa base jurídica, justificuen las iniciativas en los re-procesos de Reclutamiento y Selección del personal; la principal fuente de datos para el Reclutamiento de estos talentos los presentó el Consejo Nacional de Discapacidades -CONADIS- que hasta mediados del 2013 se encargó de la valoración del tipo, porcentaje y nivel de discapacidad, luego esta tarea pasó a ser parte de las funciones del Ninisterio de Salud Pública, ahora actores de la Autoridad Sanitaria Nacional, quien realiza la valoración de discapacidades para quien lo hace por primera vez, y de re-calificación para quien ya posee el Carnet de Discapacidad. Se dejó instituida una estrecha relación laboral social, en donde apareció: el mercado laboral que proponian las empresas que requerian la cobertura de sus vacantes, y por otro lado el mercado de recursos humanos que esbozaba perfiles técnicos ! personas que anhelaban a ocupar por méritos propios estas vacantes, antes retadas.

Además las empresas, acrecentaron métodos interesantes de captación con la Trabajadora Social y los profesionales del Dispensario Médico, apoyando la gestión del Seleccionador, iniciando campañas en la búsqueda de familiares de los empleados activos que posean un nivel de cliscapacidad, para dar cumplimiento al $\Lambda \mathrm{rt} .48$ que habla de los Sustitutos "Las y' los parientes hasta cuarto grado de consanguineidad y segundo de afinidad, cónyuge, pareja en unión de hecho, representante legal o las personas que tengan bajo su responsabilidad y/o cuidado a una persona con discapacidad severa, podrán formar parte del porcentaje de cumplimiento laboral, de conformidad con el reglamento. Este beneficio no podrá trasladarse a más de una (1) persona por persona con discapacidad....Prosigue: ...Se considerarán como sustitutos a los padres de las niñas, niños o adolescentes con discapacidad o a sus representantes legales. De existir otros casos de solidaridad humana, la autoridad nacional encargada de la inclusión económica y social validará al sustituto, de conformidad al reglamento... Adiciona además una limitante: Las y los empleadores no podrán contratar más del cincuenta por ciento $(50 \%)$ de sustitutos del porcentaje legal establecido". Siendo de gran ayuda al cumplimiento de la ley, por lo que también es un aliciente para la preocupación familiar de los empleados, esto consuela de alguna forma a la responsabilidad de enfrentar una situación de esta naturaleza para quien la padece en su núcleo familiar, en el área económica sobre todo. 
De la misma forma a los Seleccionadores del personal les aruda entonces a buscar entre los empleados que padecen de alguna discapacidad no tan obvia, pero no por eso menos cierta a que se sometan a los exámenes de rigor para precisar su situación. $O$ de quien haga de sustituto para cumplir con el porcentaje establecido por la ler: Como se ve estos artículos han renido al auxilio de las empresas para la observancia de la ley laboral porque el Reclutamiento y la Captación es sólo el punto de partida para la selección del personal: porque una vez que concurran los aplicantes en mención existe la posibilidad de caer en la impulso de seleccionarlos sólo por cumplir con el porcentaje solicitado por la ley, sin tomar en cuenta el proceso de selección imparcial para que la contratación beneficie a ambos protagonistas: a los candidatos concederles un lugar de trabajo en donde se consideren útiles a tiempo completo, dándoles una satisfacción laboral y personal en su condición de discapacitante y por otro lado, la empresa tener un personal capacitado y facultado para realizar sus funciones, para que sea realmente beneficioso por sus aportes a través del acatamiento de las tareas asignadas.

Algo muy significativo que hay que recalcar en esta ley, sobre todo para afrontar de alguna forma el estado de indefensión al cual se encuentran expuestos y con ello garantizarles una tranquila estabilidad en sus jornadas laborales, por lo que está dentro de las obligaciones de los empleadores que la salida intempestiva y arbitraria de sus empleos, se vean reflejadas en sanciones económicas muy fuertes y eso lo encontramos en el Art. 51 de esta ley, que indica lo siguiente:...."en el caso de despido injustificado de una persona con discapacidad o de quien tuviere a su cargo la manutención de la persona con discapacidad, deberá ser indemnizada con un valor equivalente a dieciocho (18) meses de la mejor remuneración, adicionalmente de la indemnización legal correspondiente. Saltando un párrafo acota finalmente: ..... Además, para la supresión de puestos no se considerarán los que ocupen las personas con discapacidad o quienes tengan a su cuidado y responsabilidad un hijo, cónıuge, pareja en unión de hecho o progenitor con discapacidad, debidamente certificado por la Autoridad Sanitaria Nacional". Hoy; dentro de las estadísticas que se registran en el Ministerio de Relaciones Laborales en caso de litigios por despidos intempestivos no descansa ninguno en archiro que se haya levantado por inobservancia de esta normativa; lo mencionado antes no establece una caución que no puede darse; lo cual obliga a llevar un seguimiento de desenvolvimiento $y$ desempeño del personal; porque también es cierto que el pensamiento colectivo de la sociedad ecuatoriana en especial de aquellos que omiten el contenido de esta ley; es asumir que la contratación de una persona con condiciones discapacitantes está garantizada por su estabilidad en las organizaciones independiente de su beneficio $y$ actuación; cuando la realidad legal es que toda persona con esta condición especial o sin ella está sujeta al cumplimiento de un contrato de trabajo normal; es decir que conlleva el período de noventa días y el tiempo de plazo fijo a uno o dos años, para luego transformarse a indefinido. El artículo en mención no constituye una permanencia obligatoria que pueda saltear las normativas organizacionales. Se debe recordar que en el párrafo 3 del Artículo 47 dictamina que "el trabajo que se le asigne a una persona con discapacidad deberá ser acorde a sus capacidades, potencialidades y talentos, garantizando su integridad en el desempeño de sus labores; proporcionando los implementos teóricos y tecnológicos para su realización; y, adecuando o readecuando su ambiente o área de trabajo en la forma que posibilite el cumplimiento de sus responsabilidades laborales". Si se toma en consideración y a cabalidad estas medidas de 
selección reducen el riesgo de que se formen conflictos de esta naturaleza, sobre todo para evitar un visto bueno por negligencia manifiesta, propuesto por el empleador.

Hasta ahora se menciona los aspectos optimistas, de las Reformas Legales al Código de Trabajo para el resguardo de discapacitados en busca de su adecuada inclusión en el mundo laboral, demostrando un saldo en positivo para los mismos en orden prioritario $\mathrm{y}$, luego para las organizaciones; sin embargo hay circunstancias que quedan fuera de este saldo: la primera es que la participación de inclusión laboral no ha podido ser tratado equitativamente en las provincias del país, porque la discapacidad, sea esta física, mental o intelectual no es una condición deseable como para buscarla por decisión propia, e igualar con un número por sector, cantón o provincia del país; a lo anterior debemos sumarle que las condiciones discapacitantes, no van en concordancia con las necesidades organizacionales $\mathrm{y}$ esto es lo que ha ocasionado que los perfiles técnicos y humanos compatibles con los espacios laborales sean deseados para los contratos; como segundo $y$ punto final es necesario señalar que el probable mal manejo y errado entendimiento de la Ley Orgánica de Discapacidades por parte de sus patrocinados podría dejar una puerta abierta para la comodidad laboral, puesto que se entendería como una estabilidad laboral sin mérito alguno para merecerla, a no ser la obtención del carnet habilitante que faculta al ciudadano a gozar de sus beneficios; de darse de esta mala manera como las empresas están preparadas para dar paso a este comportamiento dañino para ellas, pero más aún para el empleado.

El cuidado hacia las personas con discapacidad en nuestro país, es considerado como uno de los problemas sociales más descuidados por el Estado a través de la historia republicana, ya que no le ha dado la importancia debida y si ha existido, no ha cubierto los intereses de este sector que bien puede ser aprovechado para mejorar en algo su calidad de vida, en cuanto a su economía particular como en su influencia en el mercado laboral y económico de nuestro país. Las pocas iniciativas rescatables han partido de alguna forma del sector privado $y$ de algunas instituciones del sector público que han asumido esa tesponsabilidad de manera más diligente, sobre todo en los últimos veinte años.

Se ha observado, que la atención hacia las personas con discapacidad en el Ecuador se inicia hace más o menos medio siglo, por iniciativa familiar o por intermedio de organizaciones privadas, pero bajo los criterios de humanidad y ayuda social, con parámetros muy elementales y no técnicos o científicos, por la importancia que esta circunstancia requiere, siendo las primeras beneficiadas las organizaciones de personas ciegas y sordas, quienes lograron organizarse poco a poco para atender sus necesidades.

El Estado ecuatoriano intercedió a través de entidades del Ministerio de Educación, instaurando escuelas de educación especial por las décadas del 40 al 60 . Y esto aconteció por las secuelas que dejó la poliomielitis que brotó en la década del 50, por lo que se fundó en Guayaquil SERLI, por el Dr. Emiliano Crespo.

En los años setenta, el estado formula políticas muy elementales para la atención de las personas con discapacidad en los campos de la educación, la salud, el bienestar social en esa época, por lo que su cobertura se amplía a la creación de varios servicios, da la pauta a pasos importantes en la organización del Estado, para atender a la discapacidad.

Por ello el año de 1973 el Estado crea el CONAREP Consejo Nacional de Rehabili- 
tación Profesional, que se le confió la formación ocupacional e inserción laboral de las personas con discapacidad. En el año de 1977 se remitió la Lẹ General de Educación en la que se señala, "que la educación especial es una responsabilidad del Estado". Otra iniciativa estatal muy importante en esta materia fue la creación de la Unidad de Educación Especial en abril de 1979.

En el año de 1980 se crea la División Nacional de Rehabilitación en el Ministerio de Salud encargándose de la organización e implementación de la Rehabilitación Funcional. Desarrollando su protección asistencial de servicios de Medicina Física en casi todas las provincias que se suman a las unidades de rehabilitación de la Seguridad Social, en los años que van desde el 1981 a 1984.

En el año de 1982 se expide la Ley de Protección del Minusváliclo, que crea la Dirección Nacional de Rehabilitación Integral del Minusváliclo-DIN $\Lambda$ RIM, y le asigna al Ministerio de Bienestar Social la orientación y' coordinación con los demás establecimientos en todo lo afín con esta actividad, y fija el desvanecimiento del CON $\Lambda$ REP.

Comienzan a surgir por esa época, intenciones privadas como lo son el Instituto Nacional del Niño y la Familia-INNFA, con la instauración de varios centros de rehabilitación y escuelas de educación especial. Y otras (ONGS que se destacaron por su labor en favor de las personas con discapacidad como son: ASENIR, FASINARM, SERLI, FUND $\Lambda C I O ́ N$ GENERAI, ECUATORIAN $\Lambda$, ADINEA, FUNDACION HERMANO MIGUEL, CEBYCAN, FUNAPACE, OLIMPIADAS ESPECIALES, entre otras. (Cazar, 1989)

İn 1989 se constituyó la Comisión Interinstitucional de Análisis de la Situación de las Discapacidades en el Ecuador (CIASDFi), la misma que impulsó el diseño y la publicación del Plan Nacional de Discapacidades (Consejo Nacional de Discapacidades, 2012, citado en Montaño, 2012), donde se trabajó mediante una labor interinstitucional con técnicos en discapacidades, delegados de los ministerios de Salud, Educación, Bienestar Social, Trabajo, el INNFA, el CONADE. Posteriormente se expidió la Ley 180 sobre Discapacidades, la cual entró en vigencia el 10 de agosto de 1992 y dispuso la creación del Consejo Nacional de discapacidades del Ecuador, también conocido como CONADIS (Consejo Nacional de Discapacidades, 2012, citado en Montaño, 2012).

In la actualidad, el CONADIS es la máxima organización del Estado encargada de tratar el tema de discapacidad (CON $\Lambda_{-}^{-}$ DIS I:CUADOR, 2008, citado en Nontaño, 2012). Intre las funciones que el CONADIS desempeña están las siguientes: determinar las políticas nacionales sobre el tema de discapacidad, fomentar su respectivo cumplimiento, aprobar y vigilar el Plan Nacional de Discapacidades e impulsar la investigación en esta área (Consejo Nacional de Discapacidades, 2012, citado en Montaño, 2012).

\section{Referencias legales que amparan a las personas con discapacidad}

\section{Constitución de la República del Ecuador (2008)}

Art. 330. Las personas con discapacidad tienen garantizada la inserción $\mathrm{J}^{\prime}$ accesibilidad en igualdad de condiciones al trabajo remnnerado. Se probibe además la reducción de la remuneración del trabajador con discapacidad.

Art. 333. El Estado promorerá un régimen laboral que raja en armonia con las necesidades del cuidado bumano, en este caso de las personas con discapacidad.

\section{Reforma del Código de Trabajo}

Es obligación del empleador público y 
privado que cuente con un minimo de 25 trabajadores, contar al menos con una persona con discapacidad para el año 2006.

$$
\begin{aligned}
& 2007=1 \% \\
& 2008=2 \% \\
& 2009=3 \% \\
& 2010=4 \%
\end{aligned}
$$

Este 4\% es fijo y se aplicará para los años posteriores.

\section{Ley Orgánica del Servicio Público}

Art. 64. Se garantiza la inserción laboral sin discriminación de manera progresiva j' basta "In 4\% del total de servidores o servidoras.

Art. 47. Ley Orgánica de Discapacidades (Registro Oficial No.796)

Art. 47.- Inclusión laboral.- La o el empleador público o privado que cuente con un número mínimo de veinticinco (25) trabajadores está obligado a contratar, un mínimo de cuatro por ciento (4\%) de personas con discapacidad, en labores permanentes que se consideren apropiadas en relación con sus conocimientos, condiciones físicas y aptitudes individuales, procurando los principios de equidad de género y diversidad de discapacidades. El porcentaje de inclusión laboral deberá ser distribuido equitativamente en las provincias del país, cuando se trate de empleadores nacionales; y a los cantones, cuando se trate de empleadores provinciales.

En los casos de la nómina del personal de las Fuerzas Armadas, Policía Nacional, Cuerpos de Bomberos y Policías Municipales del sector público, empresas de seguridad y vigilancia privada; se tomará en cuenta únicamente la nómina del personal administrativo para el cálculo del porcentaje de inclusión laboral detallado en el inciso anterior, excluyendo el desempenoo de funciones operativas en razón del riesgo que implica para integridad física de la personas con discapacidad.

El trabajo que se asigne a una persona con discapacidad deberá ser acorde a sus capacidades, potencialidades y talentos, garantizando su integridad en el desempeño de sus labores; proporcionando los implementos técnicos y tecnológicos para su realización; y, adecuando o readecuando su ambiente o área de trabajo en la forma que posibilite el cumplimiento de sus responsabilidades laborales.

En caso de que la o el empleador brinde el servicio de transporte a sus trabajadores, las unidades de transporte deberán contar con los accesos adecuados correspondientes o serán validos otros beneficios sociales de acuerdo al reglamento de la presente l.ey.

Para efectos del cálculo del porcentaje de inclusión laboral se excluirán todos aquellos contratos que la Ley de la materia no establezca de naturaleza estable o permanente.

Esta Ley motivó a que muchas personas con discapacidad se conviertan en parte de la masa laboral de nuestro país, circunstancias que se ven reflejadas en las estadísticas realizadas por el Gobierno a través de su organismo respectivo que es el Consejo Nacional de Discapacitados (CONADIS), donde se estima que por lo menos 28000 ciudadanos (2013) con diferentes discapacidades sean parte del mercado laboral actual, cifra muy significativa y referente que deja marginado el concepto social de que la incapacidad de trabajar por parte de un ciudadano que adolece alguna molestia, ya sea física o intelectual no pueda aportar de alguna manera a la economía particular o nacional. 
Sin embargo para ellos, se han establecido diversos procesos organizacionales legales propuestos al respecto, devolviéndole de esta forma a la persona con condición discapacitante, la dignidad y el espacio justo dentro de la sociedad, aun tomando en cuenta también que de alguna u otra forma su deficiencia física se traslada a la familia que lo rodea, situación que de alguna manera u otra lo reconoce muy sabiamente esta ley.

Por ello al convertirse en ley se convierte de hecho en una política pública, establecida previamente en nuestra constitución, por lo que de esta forma devuelve la dignidad laboral a ciudadanos que con una condición discapacitante aún son parte productiva de nuestro país. De esta manera Ecuador responde a un proceso que de manera paulatina y progresiva se da en el país, logrando la adecuada inserción de personas con condiciones discapacitantes en las empresas, realizando actividades diferentes; colaborando desde sus diferentes procedimientos internos al crecimiento convertidor e influyente en una sociedad más ecuánime, menos discriminatoria $y$ limitante.

Por último habria que agregar también que cualquier acto de marginación o maltrato con respecto a este grupo considerado a pesar de todo por nuestro estado, como vulnerable se encuentra debidamente protegido en el nuevo código integral penal en donde se lo tífica como Delito de odio Art. 177.- Actos de odio.- La persona que cometa actos de violencia física o psicológica de odio, contra una o más personas en razón de su nacionalidad, etnia, lugar de nacimiento, edad, sexo, identidad de género u orientación sexual, identidad cultural, estado civil, idioma, religión, ideología, condición socioeconómica, condición migratoria, discapacidad, estado de salud o portar VIH, será sancionada con pena privativa de libertad de uno a tres años.
Si los actos de violencia provocan heridas a la persona, se sancionará con las penas privativas de libertad previstas para el delito de lesiones agravadas en un tercio. Si los actos de violencia producen la muerte de una persona, será sancionada con pena privativa de libertad de veintidós a veintiséis años.

De esta forma su protección como grupo denominado también de las minorías, se encuentra amparado a la luz de la ley penal para su mayor protección, a la que de alguna manera se encuentra expuesto en la sociedad, como en el ámbito laboral ý social.

\section{Conclusiones}

La Aplicación de la Ley Orgánica de Discapacidades en el Ecuador a pesar de conseguir Reformas laborales, seguirá cumpliendo con su fin, en la medida en que la sociedad adquiera una visión inclusiva acatando los procedimientos que la facultan dentro de las organizaciones empresariales.

Hasta ahora su cumplimiento y acatamiento se ha dado por la imposición que ha ejercido esta ley a través de las sanciones ante su inobservancia; y con ello ha servido de gran ayuda la implementación de Programas de Coordinación Interdisciplinarios entre las organizaciones interesadas en su ejecución con las organizaciones tanto públicas como privadas, por lo que hoy los resultados presentados son una simbiosis social y psicológica que ha actuado efectivamente en la autoestima de las personas con condiciones discapacitantes, que ante la acogida y aceptación de sus derechos, han aprovechado la oportunidad en el espacio laboral que se les ha otorgado, siendo recíprocos en la ejecución de sus funciones, desempeñándolas de manera efectiva y productiva ayudando a generar rentabilidad para esas empresas. 
Los mecanismos de control que se han implementado para cumplir con las Reformas Laborales para la defensa de los Discapacitados, no examinaron en sus estatutos que las empresas ecuatorianas debieran contar también con un sistema formativo, cuyos proyectos aborden las necesidades educativas especiales (NEE).

No se determina los motivos por los cuales el porcentaje más alto de ocupación de empleados con condición discapacitante se desempeña en puestos de apoyos y servicios; para que en base a ello se propongan soluciones incentivadoras al crecimiento profesional de los mismos y, que además sea atractivo a las organizaciones contratantes.

También es importante anotar que, desde los inicios de la vida escolar de las personas con discapacidad se las vaya capacitando para las demandas del mundo laboral de hoy, que cada vez es más profesional y estricto. Por lo que es importante definir también mallas educativas, para reforzar también una capacitación práctica, analizada técnicamente, para aprovechar las experticias que dentro de su discapacidad, la madre naturaleza les ofrece.

Es importante detallar dentro de la ley, las garantías de estabilidad laboral para los colaboradores con condición discapacitante, porque la exagerada protección laboral podría actuar como una arma de doble filo en casos puntuales: puede incentivar a la desidia laboral, o puede generar un rechazo por parte de compañeros que lo asuman como un favoritismo o una ventaja para quien posee esta condición, causando de alguna manera el rechazo o casos de abuso o maltrato. Lo mencionado hace que la discapacidad adquiera un sentido de privilegio o de seguridad para ambos casos.

\section{Bibliografía}

Agencia pública de noticias de Ecuador y Suramérica (2009). "Ecuador impulsa políticas públicas a favor de los discapacitados", disponible en: http://andes.info. ec/sociedad/ecuador-impulsa-politicaspublicas-a-favor-de-discapacitados- 192 . html

Alles, M. (2005). Diccionario de preguntas. Gestión por competencia: cómo planificar la entrevista por competencia. ( $1^{\mathrm{a}} \mathrm{ed}$.). Buenos Aires, Argentina: Granica.

Asamblea Constituyente, Constitución del Ecuador, 2008, versión digital de:

ASAMBLEA NACIONAL, Ley' Orgánica de Servicio Público, 2010, versión digital de: http://www.mrl.gob.ec/index. php?option $=$ com_content\&view $=$ article \&id $=276 \&$ Itemid $=233$

Cazar, R. (1989). Breve análisis de la situación de las discapacidades en el Ecuador. Quito, Ecuador: Conadis/ CIASE.

Congreso Nacional de la República del Ecuador. (1992). Código de menores; ley del minusválido; reglamento de adopción de menores; reglamento de aplicación de beneficios tributarios al minusválido. Quito, Ecuador: Corporación de Estudios y Publicaciones.

Consejo Nacional de Igualdad de Discapacidades (CONADIS), "II Plan Nacional de Discapacidades", Gobierno Nacional del Ecuador, Quito, Noviembre 2005, p. 13.

Consejo Nacional de Igualdad de Discapacidades (CONADIS), "II Plan Nacional de Discapacidades", Gobierno Nacional del Ecuador, Quito, noviembre 2005, p. 13. 
Consejo Nacional para las personas con discapacidad. Personalidades $y$ la discapacidad: perseverancia comprobada. En: http://www.conapdis.gob.ve/index. $\mathrm{php} /$ protagonistas/615-personalidadesy-la-discapacidad-perseverancia-comprobada

Constitución de la República del Ecuador. 2008

Diario Hoy: (24 de febrero de 2012). Inclusión laboral tuvo reconocimiento en Quito. EL HOY.

Doron, R. y Parot, F. (2008). Diccionario Akal de Psicología. Madrid, España: Ediciones $\mathrm{Akal}$.

Ecuador: La Discapacidad en Cifras. (2005). Quito.

Escobar, G. (2008), "Situación de las Personas con Discapacidad en Iberoamérica", Federación Iberoamericana del Ombudsman (FIO), p.53.

FENEDIF y AECID, Convenio de cooperación del proyecto Servicio de Inserción Laboral de Personas con Discapacidad en Ecuador: Trabajando por la Inclusión, 2009, versión digital de:

Groce N., Gannotti M. (2008), "Marginados de la sociedad: los discapacitados de América Latina", The International Development Research Centre, pp. 18-20.

Grof, S. (2003). La evolución de la conciencia. Barcelona, España: Kairós.

HoyQuito. (2007, 27 de diciembre). Empresas deben incluir a discapacitados. Hoy Quito. Recuperado el 1 de agosto de 2014, de http://www.hoy.com.ec/noticias-ecuador/empresas-deben-incluir-a- discapacitados-285587.html http://www.asambleanacional.gov.ec/ documentos/Constitucion-2008.pdf http://www.discapacidadesecuador.org/ portal/images/stories/File/Proyecto $\% 20$ AECID $\% 20$ COCENFE/proyecto $\% 20$ aecid $\% 20$ cocemfe.pdf http://www.vicepresidencia.gob.ec/programas/manuelaespejo/mision

La Ley Orgánica de Discapacidades, 25 de septiembre del 2012.Registro Oficial No. 796

López, R., Programas para la Orientación Vocacional y Laboral, curso de la maestría en Educación Especial, mención en Educación de las Personas con Discapacidad Visual, UPS, Quito, 2008.

Maldonado, S. (2005), "¿Exclusión o Discriminación? El Caso de las Personas con Discapacidad en el Mercado Laboral Peruano", Observatorio de la Economía Latinoamericana, pp.14.

Martinez, P. Z., Fernández, C. D. T., \& Garcia, F. E. (2013). Una propuesta para contribuir al desarrollo humano. El Periplo Sustentable, 14(24).

Montaño, C. (2012). Determinación y evaluación de estereotipos mantenidos hacia las personas con discapacidad física en el Ecuador.

Moreno, L. (2007), "Programa Ecuador sin Barreras", disponible en: http://www. vicepresidencia.gob.ec/programas/ecuadorsinbarreras/ecuador-sin-barreras www.mintrab.gov.ec

Moreno, L. (2009), "Misión Manuela Espejo", Vicepresidencia de la República del Ecuador. 
Pastore, J. (2008), "Oportunidades laborales para las personas con discapacidad", Universidad de San Pablo, Río de Janeiro, disponible en: http://www.disabilityworld. org/09-10_01/spanish/trabajo/brazil. shtml

Pintos, E. (1996). Psicología transpersonal: conciencia y meditación. ( $1^{\mathrm{a}}$ ed.). México: Plaza y Valdés.

Vicepresidencia de la República. (21 de mayo de 2012). Ecuador de Inmediato. Obtenido de http://www.ecuadorinmediato.com/Noticias/news_user_view/ mas_de_300_mil_personas_con_discapacidad_fueron_beneficiadas_en_ecuador_afirmo_lenin_moreno_en_ginebra--173872

Villacís, B. (3 de marzo de 2010). Byron Villacís: censo nacional es una necesidad civica más que política. (E. Inmediato, Entrevistador)

Vos, R. (2000), "Ecuador 1999: Crisis económica y protección social", Estudios e informes del SIISE, Quito-Ecuador, p.48.
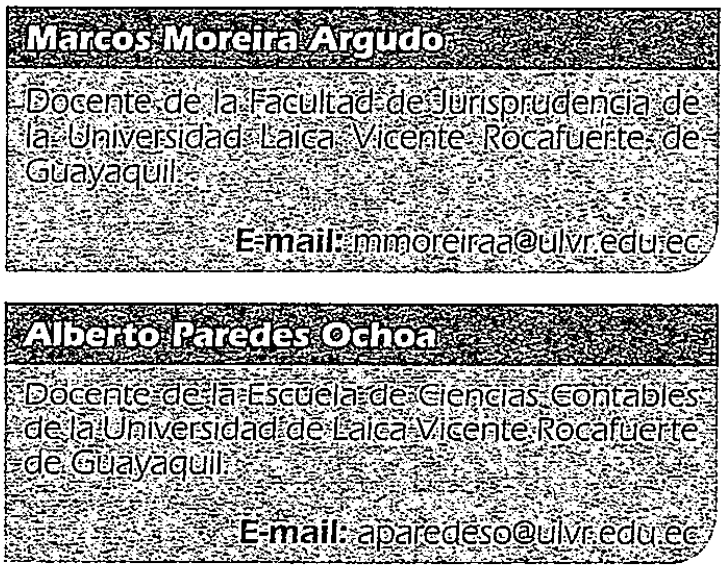
' 